\title{
THE CO-RANK OF THE FUNDAMENTAL GROUP: THE DIRECT PRODUCT, THE FIRST BETTI NUMBER, AND THE TOPOLOGY OF FOLIATIONS
}

\author{
IRINA GELBUKH
}

\begin{abstract}
We study $b_{1}^{\prime}(M)$, the co-rank of the fundamental group of a smooth closed connected manifold $M$. We calculate this value for the direct product of manifolds. We characterize the set of all possible combinations of $b_{1}^{\prime}(M)$ and the first Betti number $b_{1}(M)$ by explicitly constructing manifolds with any possible combination of $b_{1}^{\prime}(M)$ and $b_{1}(M)$ in any given dimension. Finally, we apply our results to the topology of a Morse form foliations. In particular, we construct a manifolds $M$ and a Morse form $\omega$ on it for any possible combination of $b_{1}^{\prime}(M), b_{1}(M), m(\omega)$, and $c(\omega)$, where $m(\omega)$ is the number of minimal components and $c(\omega)$ is the maximum number of homologically independent compact leaves of $\omega$.
\end{abstract}

\section{INTRODUCTION AND MAIN RESULTS}

Co-rank of a group $G$, also known as inner rank, is the maximum rank of a free homomorphic image of $G$. In a sense, co-rank is a notion dual to the rank; unlike rank, co-rank is algorithmically computable for finitely presented groups. This notion has been re-invented various times in different branches of mathematics, and its properties relevant for the corresponding particular task have been studied in different contexts. The notion of co-rank, called there inner rank, was apparently first mentioned in [20] in the context of solving equations in free groups. Co-rank of the free product of groups was calculated using geometric [15] and algebraic 22] methods.

Co-rank is extensively used in geometry, especially in geometry of manifolds, as

$$
b_{1}^{\prime}(M)=\operatorname{corank}\left(\pi_{1}(M)\right),
$$

the co-rank of the fundamental group $\pi_{1}(M)$ of a manifold $M$. For example, it has been repeatedly shown to coincide with the genus $g$ of a closed oriented surface: $b_{1}^{\prime}\left(M_{g}^{2}\right)=g[21,14,8,17$.

2010 Mathematics Subject Classification. 14F35, 57N65, 57R30.

Key words and phrases. co-rank, inner rank, manifold, fundamental group, direct product, Morse form foliation . 
In the theory of 3-manifolds, $b_{1}^{\prime}(M)=c(M)$ [27, 15, the cut number: the largest number $c$ of disjoint two-sided surfaces $N_{1}, \ldots, N_{c}$ that do not separate $M$, i.e., $M \backslash\left(N_{1} \cup \cdots \cup N_{c}\right)$ is connected. It is related to quantum invariants of $M^{3}$ and gives a lower bound on its Heegaard genus 11. Around 2001, J. Stallings, A. Sikora, and T. Kerler discussed a conjecture that for a closed orientable 3-manifold it holds $b_{1}^{\prime}(M) \geq \frac{b_{1}(M)}{3}$, where $b_{1}(M)$ is the Betti number. This conjecture was later disproved by a number of counterexamples, such as [12, 17. In this paper we, in particular, give a complete characterization of possible pairs $b_{1}^{\prime}(M), b_{1}(M)$ for any given $\operatorname{dim} M$.

In systolic geometry, every unfree 2-dimensional piecewise flat complex $X$ satisfies the bound $S R(X) \leq 16\left(b_{1}^{\prime}(X)+1\right)^{2}[16$, where $S R$ is the optimal systolic ratio.

In the theory of foliations, for the foliation $\mathcal{F}_{\omega}$ defined on $M \backslash \operatorname{Sing} \omega$ by a closed 1-form $\omega$ with the singular set Sing $\omega$, it was shown that if $b_{1}^{\prime}(M) \leq 1$ and codim Sing $\omega \geq 3$ with Sing $\omega$ contained in a finite union of submanifolds of $M$, then $\mathcal{F}_{\omega}$ has no exceptional leaves [18. Foliations have numerous applications in physics, such as general relativity [4, superstring theory [3, 2, etc.

The notion of co-rank of $\pi_{1}(M)$, the notation $b_{1}^{\prime}(M)$, and the term the first non-commutative Betti number were first introduced in [1 to study Morse form foliations, i.e., foliations defined by a closed 1-form that is locally the differential of a Morse function on a smooth closed manifold $M$.

A Morse form foliation can have compact leaves, compactifiable leaves and minimal components [9]. In [1], it was proved that if $b_{1}^{\prime}(M) \leq 2$, then each minimal component of $\mathcal{F}_{\omega}$ is uniquely ergodic. On the other hand, if $b_{1}^{\prime}(M) \geq 4$, then there exists a Morse form on $M$ with a minimal component that is not uniquely ergodic. If the form's rank

$$
\operatorname{rk} \omega>b_{1}^{\prime}(M)
$$

then the foliation $\mathcal{F}_{\omega}$ has a minimal component [19] here $\operatorname{rk} \omega=\operatorname{rk}_{\mathbb{Q}}[\omega]$, where $[\omega]$ is the integration map.

Though co-rank is known to be algorithmically computable for finitely presented groups [23, 26], we are not aware of any simple method of finding $b_{1}^{\prime}(M)$ for a given manifold. This value is, however, bounded by the isotropy index $h(M)$, which is the maximum rank of a subgroup in $H^{1}(M, \mathbb{Z})$ with trivial cupproduct 24. Namely, for a smooth closed connected manifold it holds [8, 5]

$$
b_{1}^{\prime}(M) \leq h(M)
$$

while for $h(M)$ there are simple estimates via $b_{1}(M)$ and $b_{2}(M)$ [25]. 
For the connected sum of $n$-manifolds, $n \geq 2$, except for non-orientable surfaces, it holds:

$$
\begin{aligned}
& b_{1}\left(M_{1} \# M_{2}\right)=b_{1}\left(M_{1}\right)+b_{1}\left(M_{2}\right), \\
& b_{1}^{\prime}\left(M_{1} \# M_{2}\right)=b_{1}^{\prime}\left(M_{1}\right)+b_{1}^{\prime}\left(M_{2}\right),
\end{aligned}
$$

which for $\operatorname{dim} M \geq 3$ follows from the Mayer-Vietoris sequence and by 22 , Proposition 6.4], respectively. Also, for the direct product the Künneth theorem gives:

$$
b_{1}\left(M_{1} \times M_{2}\right)=b_{1}\left(M_{1}\right)+b_{1}\left(M_{2}\right) .
$$

In this paper, we show that the fourth combination is very different:

$$
b_{1}^{\prime}\left(M_{1} \times M_{2}\right)=\max \left\{b_{1}^{\prime}\left(M_{1}\right), b_{1}^{\prime}\left(M_{2}\right)\right\}
$$

(Theorem 3.1), which completes the missing piece to allow calculating $b_{1}^{\prime}(M)$ for manifolds that can be represented as connected sums and direct products of simpler manifolds.

We give a complete characterization of the set of all possible combinations of $b_{1}^{\prime}(M)$ and $b_{1}(M)$ for a given $n=\operatorname{dim} M$. Namely, for $b^{\prime}, b \in \mathbb{Z}$, there exists a connected smooth closed $n$-manifold $M$ with $b_{1}^{\prime}(M)=b^{\prime}$ and $b_{1}(M)=b$ iff

$$
\begin{array}{ll}
n \geq 3: & b^{\prime}=b=0 \text { or } 1 \leq b^{\prime} \leq b ; \\
n=2: & 0 \leq b, b^{\prime}=\left[\frac{b+1}{2}\right] ; \\
n=1: & b^{\prime}=b=1 ; \\
n=0: & b^{\prime}=b=0 ;
\end{array}
$$

the manifold can be chosen orientable, except for odd $b$ when $n=2$ (Theorem 4.1). Using (1.1)-(1.2), we explicitly construct such a manifold (Construction 4.3).

We apply the obtained results to estimation of the number of minimal components $m(\omega)$ and the maximum number of homologically independent compact leaves $c(\omega)$ of the foliation $\mathcal{F}_{\omega}$ of a Morse form $\omega$ on $M$. The smaller $b_{1}^{\prime}(M)$ or $b_{1}(M)$, the more information we have about $\mathcal{F}_{\omega}$. For example,

$$
\begin{aligned}
m(\omega)+c(\omega) & \leq b_{1}^{\prime}(M)[8, \\
2 m(\omega)+c(\omega) & \leq b_{1}(M)[9 ;
\end{aligned}
$$

In particular, if $b_{1}^{\prime}(M)=0$, or, which is the same, $b_{1}(M)=0$, then all leaves of $\mathcal{F}_{\omega}$ are compact and homologically trivial and $\omega=d f$ is exact.

Theorem 4.1 which states that all combinations allowed by (1.3) are possible, implies that the two inequalities are independent. However, in special cases knowing the values of $b_{1}^{\prime}(M)$ and $b_{1}(M)$, for example, calculated using (1.1)(1.2), allows choosing one of the two inequalities as stronger. For instance, if $b_{1}^{\prime}(M) \leq \frac{1}{2} b_{1}(M)$, then the first one is stronger. 
Finally, we generalize Theorem 4.1 to a characterization of the set of possible combinations of $b_{1}^{\prime}(M), b_{1}(M), m(\omega)$, and $c(\omega)$ (Theorem 5.2). Namely, we use Construction 4.3 - the constructive proof of Theorem 4.1 - to show that (1.3) and (1.4) are the only restrictions on these four values, except for $b_{1}^{\prime}(M)=$ $\frac{1}{2} b_{1}(M)$ if $\operatorname{dim} M=2$.

The paper is organized as follows. In Section 2, we give the definitions of the Betti number $b_{1}$ and the non-commutative Betti number $b_{1}^{\prime}$ for groups and manifolds. In Section 3, we calculate $b_{1}^{\prime}\left(M_{1} \times M_{2}\right)$. In Section 4 , we describe the set of possible combinations of $b_{1}^{\prime}(M)$ and $b_{1}(M)$ for a given $\operatorname{dim} M$ and, using the results of Section 3. explicitly construct a manifold with any given valid combination of $b_{1}^{\prime}(M)$ and $b_{1}(M)$. Finally, in Section 5 we use the manifold constructed in Section 4 to describe the set of possible combinations of the number of minimal components and the maximum number of homologically independent compact leaves of a Morse form foliation.

\section{Definitions}

For a finitely generated abelian group $G=\mathbb{Z}^{n} \oplus T$, where $T$ is finite, its torsion-free rank, Prüfer rank, or first Betti number, is defined as $b_{1}(G)=$ $\operatorname{rk}(G / T)=n$. The notion of first Betti number can be extended to any finitely generated group by $b_{1}(G)=b_{1}\left(H_{1}(G)\right)=\operatorname{rk}\left(H_{1}(G) / \mathrm{T}\left(H_{1}(G)\right)\right)$, where $H_{1}(G)=$ $G^{a b}=G /[G, G]$ is the abelianization, or the first homology group, of the group $G$, and $\mathrm{T}(\cdot)$ is the torsion subgroup. In other words:

Definition 2.1. The first Betti number $b_{1}(G)$ of a finitely generated group $G$ is the maximum rank of a free abelian quotient group of $G$, i.e., the maximum rank of a free abelian group $A$ such that there exists an epimorphism $\varphi: G \rightarrow A$.

Consider a connected smooth closed manifold $M$. The first Betti number of $M$ is the torsion-free rank of its first homology group $H_{1}(M)$, i.e., of the first homology group of its fundamental group $\pi_{1}(M)$ :

$$
b_{1}(M)=b_{1}\left(\pi_{1}(M)\right) .
$$

A non-commutative analog of the Betti number can be defined as follows.

Definition 2.2. The co-rank corank $(G)$ [17, inner $\operatorname{rank} I N(G)$ 15] or $\operatorname{Ir}(G)$ 22, or first non-commutative Betti number $b_{1}^{\prime}(G)$ [1] of a finitely generated group $G$ is the maximum rank of a free quotient group of $G$, i.e., the maximum rank of a free group $F$ such that there exists an epimorphism $\varphi: G \rightarrow F$.

The notion of co-rank is in a way dual to that of rank, which is the minimum rank of a free group allowing an epimorphism onto $G$. Unlike rank, co-rank is algorithmically computable for finitely presented groups [23, 26]. 
The first non-commutative Betti number [1] of a connected smooth closed manifold $M$ is defined as the co-rank, or inner rank, of its fundamental group:

$$
b_{1}^{\prime}(M)=b_{1}^{\prime}\left(\pi_{1}(M)\right) .
$$

Note that a similar definition for higher $\pi_{k}(M)$ is pointless since they are abelian.

\section{Co-Rank of the fundamental group of the Direct PRoduct}

The Betti number $b_{1}(M)$ is linear in both connected sum and direct product. While the non-commutative Betti number $b_{1}^{\prime}(M)$ is linear in connected sum, its behavior with respect to direct product is very different:

Theorem 3.1. Let $M_{1}, M_{2}$ be connected smooth closed manifolds. Then

$$
b_{1}^{\prime}\left(M_{1} \times M_{2}\right)=\max \left\{b_{1}^{\prime}\left(M_{1}\right), b_{1}^{\prime}\left(M_{2}\right)\right\} .
$$

We will divide the proof into a couple of lemmas.

Lemma 3.2. Let $G_{1}, G_{2}$ be groups. Then any epimorphism

$$
\varphi: G_{1} \times G_{2} \rightarrow F
$$

onto a free group $F \neq \mathbb{Z}$ factors through a projection.

Proof. For $F=\{1\}$ the fact is trivial. Par abus de langage, denote $G_{1}=G_{1} \times 1$ and $G_{2}=1 \times G_{2}$, subgroups of $G_{1} \times G_{2}$. Suppose both $G_{i} \nsubseteq \operatorname{ker} \varphi$. Since $G_{1} \times G_{2}=\left\langle G_{1}, G_{2}\right\rangle$ and $\left[G_{1}, G_{2}\right]=1$ and denoting $F_{i}=\varphi\left(G_{i}\right)$, we have a free group $F=\left\langle F_{1}, F_{2}\right\rangle$ such that $\left[F_{1}, F_{2}\right]=1$ and by the condition $F_{1}, F_{2} \neq 1$.

Let $a, b \in F_{1}$ and $c \in F_{2}, c \neq 1$. Since $[a, c]=1$, we have $\langle a, c\rangle=\mathbb{Z}$ as both free and abelian, so $a, c \in\langle x\rangle$ for some $x \in F$, and similarly $b, c \in\langle y\rangle$ for some $y \in F$. Then $\langle x, y\rangle=\mathbb{Z}$ as a two-generated free group with a non-trivial relation $x^{k}=y^{l}=c \neq 1$, so $x, y \in\langle z\rangle$ for some $z \in F$. We obtain $a, b \in\langle z\rangle$; in particular, $[a, b]=1$.

Thus $F_{1}$ is abelian, and similarly $F_{2}$. Since $\left[F_{1}, F_{2}\right]=1$, we obtain that the non-trivial $F=\left\langle F_{1}, F_{2}\right\rangle$ is both free and abelian, thus $F=\mathbb{Z}$.

Remark 3.3. In fact this holds for any (infinite) quantity of factors: any epimorphism $\varphi: \times_{\alpha \in I} G_{\alpha} \rightarrow F$ onto a free group $F \neq \mathbb{Z}$ factors through the projection onto one of $G_{\alpha}$.

Lemma 3.4. Let $G_{1}, G_{2}$ be finitely generated groups. Then for the co-rank of the direct product,

$$
b_{1}^{\prime}\left(G_{1} \times G_{2}\right)=\max \left\{b_{1}^{\prime}\left(G_{1}\right), b_{1}^{\prime}\left(G_{2}\right)\right\} .
$$


Proof. Denote $G=G_{1} \times G_{2}$ and $m=\max \left\{b_{1}^{\prime}\left(G_{1}\right), b_{1}^{\prime}\left(G_{2}\right)\right\}$.

Let us show that $b_{1}^{\prime}(G) \geq m$. Without loss of generality assume $m=b_{1}^{\prime}\left(G_{1}\right)$. Consider an epimorphism $\varphi: G_{1} \rightarrow F$ onto a free group, $\operatorname{rk} F=m$. Then $\psi: G \rightarrow F$ such that $\psi\left(G_{2}\right)=1$ and $\left.\psi\right|_{G_{1}}=\varphi$ is an epimorphism, so $b_{1}^{\prime}(G) \geq m$.

Let us now show that $m \geq b_{1}^{\prime}(G)$. Consider an epimorphism $\varphi: G \rightarrow F$ onto a free group, rk $F=b_{1}^{\prime}(G)$. If $\varphi\left(G_{2}\right)=1$, then $\left.\varphi\right|_{G_{1}}$ is an epimorphism and thus $m \geq b_{1}^{\prime}\left(G_{1}\right) \geq \operatorname{rk} F=b_{1}^{\prime}(G)$, and similarly if $\varphi\left(G_{1}\right)=1$.

Otherwise $\left.\operatorname{rkim} \varphi\right|_{G_{i}} \geq 1$, so $m \geq b_{1}^{\prime}\left(G_{i}\right) \geq 1=b_{1}^{\prime}(G)$ by Lemma 3.2.

of Theorem 3.1. For smooth connected manifolds $M_{i}$, we have

$$
\pi_{1}\left(M_{1} \times M_{2}\right)=\pi_{1}\left(M_{1}\right) \times \pi_{1}\left(M_{2}\right),
$$

and the desired fact is given by Lemma 3.4

Example 3.5. For a torus $T^{n}=\times_{i=1}^{n} S^{1}$, we have $b_{1}^{\prime}\left(T^{n}\right)=b_{1}^{\prime}\left(S^{1}\right)=1$. Since $\pi_{1}\left(T^{n}\right) \neq\{0\}$ is free abelian, this also follows from Definition 2.2.

For the Kodaira-Thurston manifold $K T^{4}=H^{3} \times S^{1}$, we have $b_{1}^{\prime}\left(K T^{4}\right)=1$ since for the Heisenberg nil manifold, $b_{1}^{\prime}\left(H^{3}\right)=1$. This also follows from the fact that $K T^{4}$ itself is a nil manifold.

In Section 4, we will use Theorem 3.1 to explicitly construct a manifold with arbitrary given $b^{\prime}(M)$ and $b(M)$.

\section{Relation Between $b_{1}^{\prime}(M)$ And $b_{1}(M)$}

As an application of Theorem 3.1 in this section we show that there are no non-obvious relations between $b_{1}^{\prime}(M)$ and $b_{1}(M)$, and explicitly construct a manifold with any given valid pair of $b_{1}^{\prime}(M)$ and $b_{1}(M)$.

For a sphere and for low-dimensional manifolds, such as closed orientable surface $M_{g}^{2}=\#^{g} T^{2}$ and closed non-orientable surface $N_{h}^{2}=\#^{h} \mathbb{R} P^{2}, h \geq 1$, the values of $b_{1}^{\prime}(M)$ and $b_{1}(M)$ are obvious or well known:

$$
\begin{array}{lll}
\text { point: } & b_{1}^{\prime}(*)=0, & b_{1}(*)=0 ; \\
\text { circle: } & b_{1}^{\prime}\left(S^{1}\right)=1, & b_{1}\left(S^{1}\right)=1 ; \\
\text { sphere, } n \geq 2: & b_{1}^{\prime}\left(S^{n}\right)=0, & b_{1}\left(S^{n}\right)=0 ; \\
\text { orientable surface: } & b_{1}^{\prime}\left(M_{g}^{2}\right)=g, & b_{1}\left(M_{g}^{2}\right)=2 g ; \\
\text { non-orientable surface: } & b_{1}^{\prime}\left(N_{h}^{2}\right)=\left[\frac{h}{2}\right], & b_{1}\left(N_{h}^{2}\right)=h-1 .
\end{array}
$$

The value of $b_{1}^{\prime}\left(M_{g}^{2}\right)$ was calculated in [8] and [17. Lemma 2.1]. It can be also obtained as the cut-number [15, Theorem 2.1], which for a surface is the number of handles, since each non-separating two-sided circle identifies the edges of two holes. In particular, $b_{1}^{\prime}\left(N_{h}^{2}\right)$ is a sphere with $\left[\frac{h}{2}\right]$ inverted handles plus a Möbius strip for odd $h$. 
In general,

$$
0 \leq b_{1}^{\prime}(M) \leq b_{1}(M)
$$

with

$$
b_{1}^{\prime}(M)=0 \text { iff } b_{1}(M)=0,
$$

and thus

$$
b_{1}(M)=1 \text { implies } b_{1}^{\prime}(M)=1 .
$$

Indeed, since for a free group $F$ it holds $\operatorname{rk} F^{a b}=\operatorname{rk} F$ and a group epimorphism $G \rightarrow F$ induces an epimorphism $G \rightarrow F^{a b}$, it holds

$$
0 \leq b_{1}^{\prime}(G) \leq b_{1}(G)
$$

and since $\mathbb{Z}$ is both free and free abelian, $b_{1}^{\prime}(G)=0$ iff $b_{1}(G)=0$.

There are no relations between $b_{1}^{\prime}(M)$ and $b_{1}(M)$ other than (4.1)-(4.3):

Theorem 4.1. Let $b^{\prime}, b, n \in \mathbb{Z}$. There exists a connected smooth closed $n$ manifold $M$ with $b_{1}^{\prime}(M)=b^{\prime}$ and $b_{1}(M)=b$ iff

$$
\begin{array}{ll}
n \geq 3: & b^{\prime}=b=0 \text { or } 1 \leq b^{\prime} \leq b ; \\
n=2: & 0 \leq b, b^{\prime}=\left\lfloor\frac{b+1}{2}\right\rfloor ; \\
n=1: & b^{\prime}=b=1 ; \\
n=0: & b^{\prime}=b=0 ;
\end{array}
$$

the manifold can be chosen orientable iff $n \neq 2$ or $b$ is even.

Proof. For $n \leq 2$ and for $b=0$ the facts are given in (4.1), so let $n \geq 3$ and $b \geq 1$.

For $n \geq 4$, every finitely presented group is the fundamental group of a connected smooth closed orientable $n$-manifold $M$, while by [6, Theorem 3] there exists such a group $G$ with $b_{1}^{\prime}(G)=b^{\prime}$ and $b_{1}(G)=b$, which proves the result for $n \geq 4$.

Finally, let $n=3$. For any given $b \geq 1$, Harvey [12, Theorem 3.1] constructed a smooth closed orientable hyperbolic 3 -manifold $H_{b}$ with the largest possible gap between $b_{1}^{\prime}$ and $b_{1}$ :

$$
b_{1}^{\prime}\left(H_{b}\right)=1, b_{1}\left(H_{b}\right)=b .
$$

For $1 \leq b^{\prime} \leq b$, choose $k_{i} \geq 1$ such that $\sum_{i=1}^{b^{\prime}} k_{i}=b$. By (1.1), for

$$
M=\underset{i=1}{b^{\prime}} H_{k_{i}}
$$

we have $b_{1}^{\prime}(M)=b^{\prime}, b_{1}(M)=b$. 
Using Theorem 3.1, we can generalize any specific example with given $b_{1}^{\prime}(M)$ and $b_{1}(M)$ to higher dimensions, as well as to increase the gap between $b_{1}^{\prime}(M)$ and $b_{1}(M)$ :

Lemma 4.2. Let $b_{1}^{\prime}\left(M^{n}\right) \neq 0$. Then for $M^{n+k}=M^{n} \times S^{k}$ it holds

$$
\begin{array}{lll}
k=1: & b_{1}^{\prime}\left(M^{n+k}\right)=b_{1}^{\prime}\left(M^{n}\right), & b_{1}\left(M^{n+k}\right)=b_{1}\left(M^{n}\right)+1 ; \\
k \geq 2: & b_{1}^{\prime}\left(M^{n+k}\right)=b_{1}^{\prime}\left(M^{n}\right), & b_{1}\left(M^{n+k}\right)=b_{1}\left(M^{n}\right) .
\end{array}
$$

This allows us to explicitly construct a manifold with given $b_{1}^{\prime}(M)$ and $b_{1}(M)$ of any given dimension, thus giving a simple constructive proof of Theorem 4.1 for $\operatorname{dim} M \geq 3$ :

Construction 4.3. For any given $b^{\prime}, b, n \in \mathbb{Z}$ such that $b^{\prime}=b=0$ or $1 \leq b^{\prime} \leq b$, and $n \geq 3$, the following connected smooth closed oriented $n$-manifold $H_{b^{\prime}, b}^{n}$ has

$$
b_{1}^{\prime}\left(H_{b^{\prime}, b}^{n}\right)=b^{\prime}, b_{1}\left(H_{b^{\prime}, b}^{n}\right)=b .
$$

For $b^{\prime}=b=1$, consider

$$
H_{1,1}^{n}=S^{1} \times S^{n-1}
$$

and for $b \geq 2$, generalize (4.6) to higher dimensions using Lemma 4.2.

$$
H_{1, b}^{n}= \begin{cases}H_{b} & \text { for } n=3, \\ H_{b-1} \times S^{1} & \text { for } n=4, \\ H_{b} \times S^{n-3} & \text { for } n \geq 5 .\end{cases}
$$

Finally, as in (4.7), choose $k_{i} \geq 1$ such that

$$
\sum_{i=1}^{b^{\prime}} k_{i}=b
$$

and take

$$
H_{b^{\prime}, b}^{n}=\underset{i=1}{b^{\prime}} H_{1, k_{i}}^{n}
$$

By Theorem 4.1 in (4.2) both the lower bound (except for $n=1$ ) and the upper bound (except for surfaces other than $S^{2}, \mathbb{R} P^{2}$, and the Klein bottle) are exact for any given $n$. Both conditions (4.3) are impossible for $n=1$ and both conditions (4.4) are impossible for $n=0$.

In particular, the lower bound in (4.2) is achieved on $S^{n}$, while (4.8) and (4.9) provide the lower bound in the inequality in (4.5). The upper bound $b_{1}^{\prime}(M)=$ $b_{1}(M)$ for $n \geq 3$ is provided by (4.11) with $b^{\prime}=b$ :

$$
H_{b, b}^{n}=\underset{i=1}{\#}\left(S^{1} \times S^{n-1}\right) .
$$


In general, $b_{1}^{\prime}(M)=b_{1}(M)$ iff some (and thus any) epimorphism

$$
\pi_{1}(M) \rightarrow H_{1}(M) / \mathrm{T}\left(H_{1}(M)\right)
$$

factors through a free group; $\mathrm{T}(\cdot)$ is the torsion subgroup:

Proposition 4.4. For any group $G$, the following conditions are equivalent:

(i) $b_{1}^{\prime}(G)=b_{1}(G)$,

(ii) there exists an epimorphism

$$
h: G \rightarrow \mathbb{Z}^{b_{1}(G)}=H_{1}(G) / T
$$

that factors through a free group; $T \subset H_{1}(G)$ is the torsion subgroup,

(iii) any such epimorphism factors through a free group.

Proof. (i) $\Rightarrow$ (ii) Let $\varphi: G \rightarrow F$ be the epimorphism from the definition of $b_{1}^{\prime}(G)$ and $\psi: F \rightarrow F /[F, F]=\mathbb{Z}^{b_{1}^{\prime}(G)}=\mathbb{Z}^{b_{1}(G)}$ be the natural epimorphism; then $h=\psi \circ \varphi$ has the desired properties.

(ii) $\Rightarrow$ (i) Let $G \rightarrow F \rightarrow H_{1}(G) / T$ be a factorization of $h$ through a free group $F$. Then $b_{1}(G)=\operatorname{rk}\left(H_{1}(G) / T\right) \leq \operatorname{rk} F \leq b_{1}^{\prime}(G)$. By [6, Theorem 3], $b_{1}^{\prime}(G) \leq b_{1}(G)$, so we obtain $b_{1}^{\prime}(G)=b_{1}(G)$.

(ii) $\Rightarrow$ (iii) For any epimorphisms $\varphi, h: G \rightarrow H$ there exists an automorphism $\psi: H \rightarrow H$ such that $\varphi=\psi \circ h$.

(iii) $\Rightarrow$ (ii) The composition of natural epimorphisms

$$
G \rightarrow H_{1}(G)=G /[G, G] \rightarrow H_{1}(G) / T=\mathbb{Z}^{b_{1}(G)}
$$

is an epimorphism.

\section{Application to foliation topology}

The gap between $b_{1}^{\prime}(M)$ and $b_{1}(M)$ plays a role in foliation topology.

5.1. Useful facts about Morse form foliations. Consider a connected closed oriented $n$-manifold $M$ with a Morse form $\omega$, i.e., a closed 1 -form with Morse singularities - locally the differential of a Morse function. The set of its singularities Sing $\omega$ is finite. This form defines a foliation $\mathcal{F}_{\omega}$ on $M \backslash \operatorname{Sing} \omega$.

Its leaves $\gamma$ can be classified into compact, compactifiable $(\gamma \cup \operatorname{Sing} \omega$ is compact), and non-compactifiable. The set covered by all non-compactifiable leaves is open and has a finite number $m(\omega)$ of connected components, called minimal components [1. Each non-compactifiable leaf is dense in its minimal component [13. A foliation is called minimal if all its leaves are non-compactifiable, i.e., the whole $M \backslash$ Sing $\omega$ is one minimal component.

Any compact leaf has a cylindrical neighborhood consisting of leaves that are diffeomorphic and homotopically equivalent to it [10. Denote by $H_{\omega} \subseteq$ $H_{n-1}(M)$ the group generated by the homology classes of all compact leaves of $\mathcal{F}_{\omega}$. Since $M$ is closed and oriented, $H_{n-1}(M)$ is finitely generated and free; 


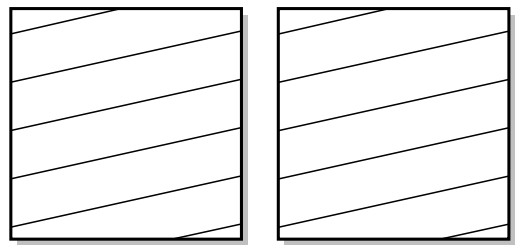

(a)

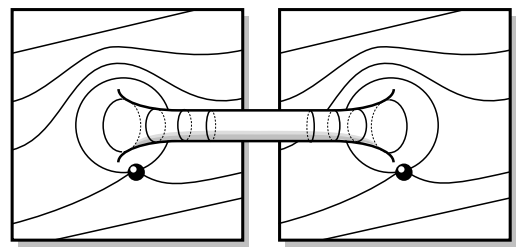

(b)

Figure 1. Construction of the form on the connected sum. (a) Manifolds $M_{1}$ and $M_{2}$ with Morse forms $\omega_{1}, \omega_{2}$, respectively. (b) A center and a conic singularity are locally added to each form, and the manifolds are glued together by spheres around the removed centers. The new form $\omega$ on $M_{1} \# M_{2}$ has two additional conic singularities.

therefore so is $H_{\omega}$. By [7, Theorem 4], in $H_{\omega}$ there exists a basis consisting of homology classes of leaves, i.e., $\mathcal{F}_{\omega}$ has exactly $c(\omega)=\operatorname{rk} H_{\omega}$ homologically independent compact leaves.

Lemma 5.1. Let $\omega_{1}, w_{2}$ be Morse forms defined on smooth closed oriented manifolds $M_{1}, M_{2}$, respectively. Then on $M=M_{1} \# M_{2}$ there exists a Morse form $\omega$ with $m(\omega)=m\left(\omega_{1}\right)+m\left(\omega_{2}\right)$ and $c(\omega)=c\left(\omega_{1}\right)+c\left(\omega_{2}\right)$.

Proof. Consider a form $\omega$ constructed as shown in Figure 1 It coincides with $\omega_{i}$ outside a small area where $M_{i}$ are glued together. We assume that each $\omega_{i}$ was locally distorted either in a minimal component or in a cylindrical neighborhood covered by homologous compact leaves. In the former case, since nearby leaves are dense on either side of the affected leaf, the distortion does not change the number of minimal components. In the latter case, even though the distortion "destroys" one compact leaf, the nearby leaves contribute the same value to $H_{\omega_{i}}$. In either case, the new compact leaves introduced in the process are homologically trivial.

Since the two sides are separated by compact leaves, each minimal component of $\omega$ lies either in $M_{1}$ or in $M_{2}$, and thus $m(\omega)=m\left(\omega_{1}\right)+m\left(\omega_{2}\right)$. Similarly, homologically non-trivial leaves of $\omega$ are homologous to either leaves of $\omega_{1}$ or leaves of $\omega_{2}$; in particular, $H_{\omega}=H_{\omega_{1}} \oplus H_{\omega_{2}}$ and thus $c(\omega)=c\left(\omega_{1}\right)+c\left(\omega_{2}\right)$.

5.2. Relation between $b_{1}^{\prime}(M), b_{1}(M), m(\omega)$, and $c(\omega)$. Let $\omega$ be a Morse form on a smooth closed orientable manifold $M, \operatorname{dim} M \geq 2$, defining a foliation with exactly $c(\omega)$ homologically independent compact leaves and $m(\omega)$ minimal components. The following inequalities have been proved independently:

$$
\begin{aligned}
m(\omega)+c(\omega) & \leq b_{1}^{\prime}(M)[8, \\
2 m(\omega)+c(\omega) & \leq b_{1}(M)[9] .
\end{aligned}
$$


For some manifolds, (1.1) and Theorem 3.1 allow direct calculation of $b_{1}^{\prime}(M)$. This may allow one to choose between (5.1) and (5.2). Namely, denoting $b^{\prime}=$ $b_{1}^{\prime}(M), b=b_{1}(M)$, unless $b^{\prime}=b=0$ we have:

(i) If $b^{\prime} \leq \frac{1}{2} b$, then (5.1) is stronger;

(ii) If $\frac{1}{2} b<b^{\prime}<b$, then they are independent;

(iii) If $b^{\prime}=b$, then (5.2) is stronger.

In particular, (5.1) is always stronger for $\operatorname{dim} M=2$. However, for any $\operatorname{dim} M \geq 3$, by Theorem 4.1 all three cases are possible; in particular, there exist manifolds for which the two estimates are independent. By (4.2), the case $b^{\prime}>b$ is impossible.

More specifically, in the case (ii) if seen as conditions on $c(\omega)$ under given $m(\omega)$ and vice versa,

- (5.1) is stronger when $m(\omega)<b-b^{\prime}$ or when $c(\omega)>2 b^{\prime}-b$;

- (5.2) is stronger when $m(\omega)>b-b^{\prime}$ or when $c(\omega)<2 b^{\prime}-b$,

and they are equivalent in case of equalities.

We can generalize Theorem 4.1 to observe that there are no relations between $m(\omega), c(\omega), b_{1}^{\prime}(M)$, and $b_{1}(M)$ other than those given by (5.1), (5.2), (4.2), (4.3), and, for an orientable surface, 4.1):

Theorem 5.2. Let $n, m, c, b^{\prime}, b \in \mathbb{N}^{0}$. There exists a smooth closed connected oriented $n$-manifold $M$ with $b_{1}^{\prime}(M)=b^{\prime}$ and $b_{1}(M)=b$, and a Morse form foliation $\mathcal{F}_{\omega}$ on it with $m$ minimal components and exactly c homologically independent compact leaves, iff

$$
\begin{array}{lll}
n=2: & \text { (4.1) for } M_{g}^{2}: & 0 \leq b=2 b^{\prime}, \\
& & 0 \leq m+c \leq b^{\prime}, \\
n \geq 3: & \text { (5.1): (4.3): } & b^{\prime}=b=0 \text { or } 1 \leq b^{\prime} \leq b, \\
& 0 \leq m+c \leq b^{\prime}, \\
& 0 \leq 2 m+c \leq b .
\end{array}
$$

Apart from a trivial foliation on $S^{n}$, the proof is given by the following constructions.

Lemma 5.3. On $S^{k} \times S^{1}, k \geq 1$, there exists Morse form foliation with $m(\omega)=$ $0, c(\omega)=0$.

Proof. The corresponding foliations for $k=1$ and $k \geq 2$ are shown in Figures 2 and 3 respectively.

Lemma 5.4. For $0 \leq m+c \leq g$, on $M=M_{g}^{2}$ there exists a Morse form foliation with $m(\omega)=m$ and $c(\omega)=c$.

Proof. As shown in Figure 4, construct $M_{g}^{2}$ as the connected sum of 


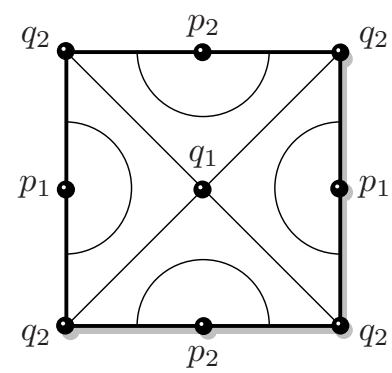

Figure 2. Torus $T^{2}$ foliated with $m(\omega)=0, c(\omega)=0$. The square is self-glued by the sides to form a torus. The foliation, shown in thin lines, has two centers $p_{1}, p_{2}$ and two conic singularities $q_{1}, q_{2}$.
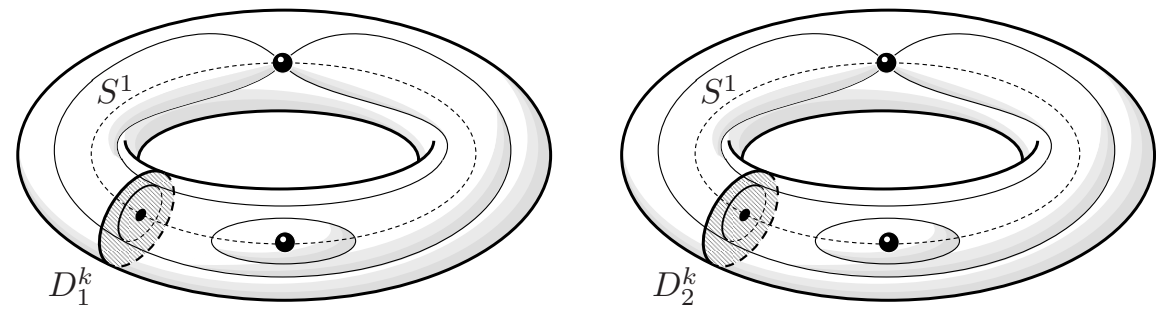

Figure 3. Manifold $S^{k} \times S^{1}, k \geq 2$, foliated with $m(\omega)=0$, $c(\omega)=0$. Shown are two manifolds $D_{i}^{k} \times S^{1}$ (solid tori for $k=2$ ), where $D_{i}^{k}$ are disks and $S^{1}$ is shown in dashed line They are glued together by the boundary, so that $D_{1}^{k} \cup D_{2}^{k}=$ $S^{k}$. The foliation, shown in thin lines, has two centers and two conic singularities, its compact leaves being either spheres $S^{k}$ or $S^{k-1} \times S^{1}$ (tori for $k=2$ ).

- $m$ tori with an irrational winding;

- $c$ tori $T^{2}$ with a compact non-singular foliation;

$-g-(c+m)$ tori with a foliation shown in Figure 2,

with the forms glued together as shown in Figure 1 Lemma 5.1 completes the proof.

This easily generalizes to higher dimensions:

Lemma 5.5. For $n \geq 3$ and $m, c, b^{\prime}, b \in \mathbb{N}^{0}$ satisfying (5.3) (5.5), there exists an n-manifold $H_{b^{\prime}, b}^{n}$ from Construction 4.3 and a Morse form on it with $c(\omega)=c$ and $m(\omega)=m$. 

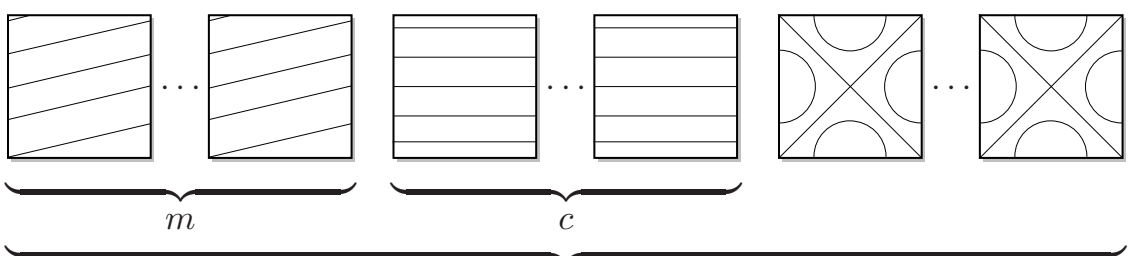

Figure 4. Construction of $M_{g}^{2}$ in the proof of Lemma 5.4 as the connected sum of tori with different foliations.

Proof. The construction is very similar to that of Lemma 5.4 Observe that in (4.10), we can choose at most $b-b^{\prime}$ summands $k_{i} \geq 2$.

If $b-b^{\prime} \geq m$ (which by (5.4) and (5.5) is always the case if $b^{\prime} \leq \frac{1}{2} b$ ), then in (4.10) choose $m$ summands $k_{i} \geq 2$, which by (5.4) leaves at least $c$ summands $k_{i}=1$ :

$$
H_{b^{\prime}, b}^{n}=\left(\begin{array}{l}
m \\
\underset{i=1}{\#}
\end{array} H_{1, k_{i}}^{n}\right) \#\left(\underset{i=1}{\stackrel{c}{\#}} H_{1,1}^{n}\right) \#\left(\# H_{1,1}^{n}\right) .
$$

Otherwise, in (4.10) choose $b-b^{\prime}$ summands $k_{i}=2$, which by (5.5) leaves

$$
b^{\prime}-\left(b-b^{\prime}\right)=b-2\left(b-b^{\prime}\right) \geq(2 m+c)-2\left(b-b^{\prime}\right)=2\left(m-\left(b-b^{\prime}\right)\right)+c
$$

summands $k_{i}=1$ :

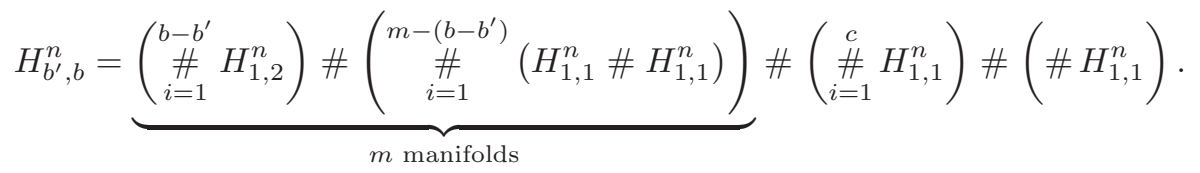

As in Lemma 5.4, the foliations on the manifolds are chosen as follows:

- On $m$ manifolds $H_{1, k_{i}}^{n}, k_{i} \geq 2$, and $H_{1,1}^{n} \# H_{1,1}^{n}$ there exists a Morse form $\omega$ with a minimal foliation [1, Theorem 1]; in particular, $m(\omega)=1$ and $c(\omega)=0$;

- On $c$ manifolds $H_{1,1}^{n}=S^{1} \times S^{n-1}$, choose a compact non-singular foliation along $S^{1}$ with leaf $S^{n-1}$;

- On the rest of $H_{1,1}^{n}$, choose a foliation shown in Figure 3

Lemma 5.1 completes the proof.

\section{REFERENCES}

[1] PIERRE ARNOUX-GILBERT LEVITT, Sur l'unique ergodicité des 1-formes fermées singulières, Invent. Math. 84 (1986), 141-156. MR Zbl 0577.58021, MR0830042

[2] ELENA MIRELA BABALIC-CALIN IULIU LAZAROIU, Foliated eight-manifolds for M-theory compactification, Journal of High Energy Physics 01 (2015), 140. 
[3] Singular foliations for M-theory compactification, Journal of High Energy Physics 03 (2015), 116.

[4] BING-LONG CHEN-PHILIPPE G. LEFLOCH, Local foliations and optimal regularity of Einstein spacetimes, J. Geom. Phys. 59 (2009), no. 7, 913-941. MR Zbl 1179.53069, MR2536854

[5] ALEXANDRU DIMCA-STEFAN PAPADIMA-ALEXANDER SUCIU, Quasi-Kähler groups, 3-manifold groups, and formality, Mathematische Zeitschrift 268 (2011), no. 1-2, 169-186. MR Zbl 1228.14018, MR2805428

[6] IRINA GELBUKH, Co-rank and Betti number of a group, Czech. Math. J. Accepted.

[7] - Presence of minimal components in a Morse form foliation, Differ. Geom. Appl. 22 (2005), 189-198. MR Zbl 1070.57016, MR2122742

[8] - Number of minimal components and homologically independent compact leaves for a Morse form foliation, Stud. Sci. Math. Hung. 46 (2009), no. 4, 547-557. MR Zbl 1274.57005, MR2654204

[9] - On the structure of a Morse form foliation, Czech. Math. J. 59 (2009), no. 1, 207-220. MR Zbl 1224.57010, MR2486626

[10] _ Close cohomologous Morse forms with compact leaves, Czech. Math. J. 63 (2013), no. 2, 515-528. MR Zbl 1289.57009, MR3073975

[11] PATRICK GILMER, Heegaard genus, cut number, weak p-congruence, and quantum invariants, J. Knot Theory Ramifications 18 (2009), no. 10, 1359-1368. MR Zbl 1181.57014, MR2583801

[12] SHELLY HARVEY, On the cut number of a 3-manifold, Geom. Topol. 6 (2002), 409-424. MR Zbl 1021.57006, MR1928841

[13] HIDEKI IMANISHI, On codimension one foliations defined by closed one forms with singularities, J. Math. Kyoto Univ. 19 (1979), 285-291.

[14] WILLIAM JACO, Heegaard splittings and splitting homomorphisms, Trans. AMS 146 (1969), 365-375. MR Zbl 0199.58601, MR0253340

[15] - Geometric realizations for free quotients, J. Austral. Math. Soc. 14 (1972), 411418. MR Zbl 0259.57004, MR0316571

[16] MIKHAIL KATZ-YULI RUDYAK-STEPHANE SABOURAU, Systoles of 2complexes, Reeb graph, and Grushko decomposition, International Math. Research Notices 2006 (2006), 1-30. MR Zbl 1116.57001, MR2250017

[17] CHRISTOPHER J. LEININGER-ALAN W. REID, The co-rank conjecture for 3manifold groups, Algebraic and Geometric Topology 2 (2002), 37-50. MR Zbl 0983.57001, MR1885215

[18] GILBERT LEVITT, 1-formes fermées singulières et groupe fondamental, Invent. Math. 88 (1987), 635-667. MR Zbl 0594.57014, MR0884804

[19] _ Groupe fondamental de l'espace des feuilles dans les feuilletages sans holonomie, J. Diff. Geom. 31 (1990), 711-761. MR Zbl 0714.57016, MR1053343

[20] ROGER C. LYNDON, The equation $a^{2} b^{2}=c^{2}$ in free groups, Mich. Math. J. 6 (1959), 89-95. MR Zbl 0084.02803, MR0103218

[21] _ Dependence in groups, Colloq. Math. XIV (1966), 275-283. MR Zbl 0141.02102, MR0188278

[22] ROGER C. LYNDON-PAUL E. SCHUPP, Combinatorial group theory, Mathematics, Springer, Berlin, 2001. MR Zbl 0997.20037, MR1812024

[23] GENNADIY SEMENOVICH MAKANIN, Equations in a free group, Math. USSR Izvestiya 21 (1983), no. 3, 483-546. MR Zbl 0527.20018, MR0682490

[24] IRINA A. MEL'NIKOVA, A test for non-compactness of the foliation of a Morse form, Russ. Math. Surveys 50 (1995), no. 2, 444-445. MR Zbl 0859.58005, MR1339274 
[25] _ Maximal isotropic subspaces of skew-symmetric bilinear mapping, Mosc. Univ. Math. Bull. 54 (1999), no. 4, 1-3. MR Zbl 0957.57018, MR1716286

[26] ALEXANDR ALEXANDROVICH RAZBOROV, On systems of equations in a free group, Math. USSR Izvestiya 25 (1985), 115-162. MR Zbl 0579.20019, MR0755958

[27] ADAM SIKORA, Cut numbers of 3-manifolds, Trans. Amer. Math. Soc. 357 (2005), no. 5, 2007-2020. MR Zbl 1064.57018, MR2115088

E-mail address: gelbukh@member.ams.org

CiC, Instituto Politécnico Nacional, 07738, Mexico City, MeXiCO 